\title{
Shell-driven Fine Structure Transition of Core Materials in Co@Au Core-shell Nanoparticles
}

\author{
Yujun Song*, Yinghui Wang, Shaoxia Ji, Jie Ding
}

(Received 26 September 2012; accepted 26 November 2012; published online 6 December 2012)

\begin{abstract}
Co@Au core shell nanoparticles (NPs) of different shell thicknesses were fabricated by a combination of the displacement process and the reduction-deposition process in a microfluidic reactor. The effect of the shell thickness on the fine structures (local atom arrangement) of core materials was investigated by X-ray Absorption Near Edge Structure (XANES) and Extended X-ray Absorption Fine Structure (EXAFS). The results indicate that the shell thickness affects the fine structure of the core materials by causing atomic re-arrangement between the hexagonal close pack (hcp) and the face centered cubic (fcc) structure, and forming $\mathrm{Co}-\mathrm{Au}$ bonds in the core-shell interface.
\end{abstract}

Keywords: Nanoparticle; Core-shell; Fine Structure; Microfluidic

Citation: Yujun Song, Yinghui Wang, Shaoxia Ji and Jie Ding, "Shell-driven Fine Structure Transition of Core Materials in Co@Au Core-shell Nanoparticles", Nano-Micro Lett. 4 (4), 235-242 (2012). http://dx.doi. org $/ 10.3786 / \mathrm{nml}$.v4i4.p235-242

\section{Introduction}

A currently active topic has been focused on the development of multi-component hybrid nanomaterials that can produce multi-mode functions or properties not available in single-component materials [1-6]. Among them, hybrid structures with magnetic components (e.g., $\mathrm{Co}, \mathrm{Fe}, \mathrm{Ni}$, iron oxides) and optical components (e.g., noble metals, semiconductors) are of much interest since these kinds of structures can endow nanomaterials with unique magnetic, optical, electronic, catalytic and other physiochemical properties in single nanoparticles (NPs) [1, 7-12]. This kind of hybrid structures will be useful for diverse applications, such as magnetic manipulation and label-free diagnosis and therapy [1, 7-12]. Their optical and magnetic properties significantly depend on core sizes and shapes, shell thickness, crystal structures of cores and shells, and the interfacial junctions between cores and shells [7-10, 12-14].

Many methods have been developed to fabricate the desired core-shell structures, such as displacement method [10, 16], redox-transmetalation process [17], the seeding-reduction process [18], direct metal salt reduction process [19-21], and the vapor deposition process $[9,22]$. However, large scale preparation of coreshell NPs with uniform, thickness-controlled continuous shells is still a challenge, particularly for those nanomaterials whose shell components are not compatible with core components [23]. This challenge not only presents a barrier for the application of these nanomaterials, but also makes it difficult to investigate the structure dependent physicochemical properties at nanoscale. In addition, characterization of the core and shell microstructure and the related fine structure transition caused by the core and shell remain open fundamental issues, which directly determines the novel discovery on the interaction between their hybrid structures and the related physiochemical properties [20, 24]. Therefore, new methods are still in development for the controlled fabrication of the core-shell NPs and the precise investigation of the relation between the core and shell structures and the physiochemical properties of these hybrid NPs is critical to facilitate their future application.

School of Materials Science and Engineering, Beihang University, Beijing 100191, China

*Corresponding author. E-mail: songyj@buaa.edu.cn 
Recently, microfluidic processes have gained much attention in the preparation of specific materials due to its in-situ spatial and temporal control of reaction kinetics, in addition to efficient mass and heat transfer [25-30]. In the nanoparticle (NP) synthesis, microfluidic reactors have been used successfully to control size, shape and crystal structure [25, 28, 31, 32], and the growth of nanoparticles [33] and to investigate the NP formation mechanism $[25,26,34,35]$. In this article, a sequential microfluidic process is developed for the synthesis of core-shell Co@Au NPs with controlled shell thickness via a combination of the displacement method and the reduction-deposition process. Our previous article has shown that the shell thickness can cause significant magnetic and optical property changes in the $\mathrm{Co@Au} \mathrm{nanoparticles} \mathrm{[36].} \mathrm{This} \mathrm{article} \mathrm{will} \mathrm{focus} \mathrm{in}$ the shell driving fine structure transition of core materials examined by X-ray Absorption near Edge Structure (XANES) and Extended X-ray Absorption Fine Structure (EXAFS).

\section{Experimental Section}

\section{Preparation of Co NPs and Co@Au core shell NPs}

Cobalt nanoparticles were prepared by the reduction of $\mathrm{CoCl}_{2}$ (99.9\%, anhydrous) in tetrahydrofuran (THF, $99.90 \%$ pure packaged under nitrogen) using lithium hydrotriethylborate $\left(\mathrm{LiBH}\left(\mathrm{C}_{2} \mathrm{H}_{5}\right)_{3}, 1 \mathrm{M}\right.$ solution in THF) as a reducing agent and $3-(\mathrm{N}, \mathrm{N}-$ dimethyldodecylammonia)-propanesulfonate (SB12, $98 \%$ ) as a stabilizer in the microfluidic reaction process, which has been discussed extensively in our previous publications [25, 28, 31, 32, 35, 37-41]. Co@Au nanoparticles with different shell thickness were prepared by the combination of the displacement method and the reduction-deposition process using $\mathrm{KAuCl}_{4}$ as $\mathrm{Au}$ source, which was described in our recent publication. Details for these preparation processes and the sketch map of the micro fluidic reactor (Scheme S1) can be referred to the literature 25, 28, 31 and 36, and the supporting information.

\section{Characterization of Co and Co@Au core-shell nanoparticles}

The particle size and shape were characterized using transmission electron microscopy (TEM, JOEL 2010, $200 \mathrm{kV}, 0.23 \mathrm{~nm}$ ) by placing a drop of well dispersed Co NPs in oxygen free water or ethanol on a carbon-coated copper TEM grid at room temperature and allowed to dry. The nanoparticle crystal structures were characterized by Selected Area Electron Diffraction (SAED), $\mathrm{X}$-ray diffraction (XRD) and X-ray absorption near $\mathrm{K}$ edge structure (XANES) and extended X-ray absorption fine structure (EXAFS). XANES and EXAFS experiments were performed at the X-ray microprobe double crystal monochromator beamline. For the Co Kedge XANES and EXAFS measurements, the beamline monochromator was calibrated with a $7.5 \mu \mathrm{m} \mathrm{hcp}$ cobalt foil. The energy bandwidth for the excitation radiation was less than $2 \mathrm{eV}$ over the range of energies examined. Spectra were background subtracted and normalized using standard procedures [40, 41].

\section{Results and Discussion}

The seed Co nanoparticles for the shell formation were prepared by our previous well-developed microfluidic process $[25,28,31,35]$. In order to increase the crystallinity of the seed NPs, the reaction was performed at $50^{\circ} \mathrm{C}$ at a low flow rate of $0.08 \mathrm{ml} / \mathrm{min}$. The size of the Co nanoparticles according to their TEM images (one shown in Fig. 1-i-a) was estimated as 4.0 $\pm 1.1 \mathrm{~nm}$, slightly larger than our previous NPs $(3.5 \pm$ $0.7 \mathrm{~nm}$ ) synthesized at room temperature [31]. Some NPs clearly show their crystal lattice fringes (Fig. 1-ib). One of them gives a lattice parameter of $2.19 \AA$, near to the d-spacing of the [100] plane of hcp cobalt [31]. Comparing with previous results on the Co NPs synthesis at room temperature [31], it is clear that the elevated temperature $\left(50^{\circ} \mathrm{C}\right)$ contributes to the increased crystallinity of the hcp cobalt NPs. The selected area electron diffraction (SAED) of the Co NPs (Fig. 1-i-c) suggests that the Co NPs preserve hcp crystal phase, with the 7 typical dotted rings from inner to outer indicating the [100], [002], [101], [102], [110], [103] and [112] crystal planes, giving d-spacing of 2.176 $\AA, 2.055 \AA, 1.858 \AA, 1.476 \AA, 1.254 \AA, 1.173 \AA$ and $1.045 \AA$, respectively. The hcp crystal structure can be further confirmed by Bragg angles at $41.86^{\circ}, 44.98^{\circ}$, $63.68^{\circ}, 76.36^{\circ}$ and $91.94^{\circ}$ in their XRD pattern (Fig. S1 in supporting information), representing the reflection from [100], [002], [102], [110] and [112] planes. These peaks give d-spacing of $2.157 \AA, 2.015 \AA, 1.461 \AA, 1.247$ $\AA$ and $1.072 \AA$ for these planes, close to those obtained by their SAED pattern (Fig. 1-i-c). In addition, the magnetic hysteresis loops at $10 \mathrm{~K}$ and $300 \mathrm{~K}$ for this kind of NPs that has been discussed in our published article show symmetric coercivities without significant exchange bias fields [36], indicating that these Co NPs are free of oxidization or with a very trace of $\mathrm{CoO}$ in NPs [44-46]. This result suggests that the SAED pattern can give enough information to identify the crystal structure of the synthesized NPs. The crystal structure identification for the core-shell NPs were characterized mainly using SAED in this article.

The TEM images give the NPs averaged diameter of $3.9 \pm 1.0 \mathrm{~nm}$ for first batch Co@Au NPs formed by 

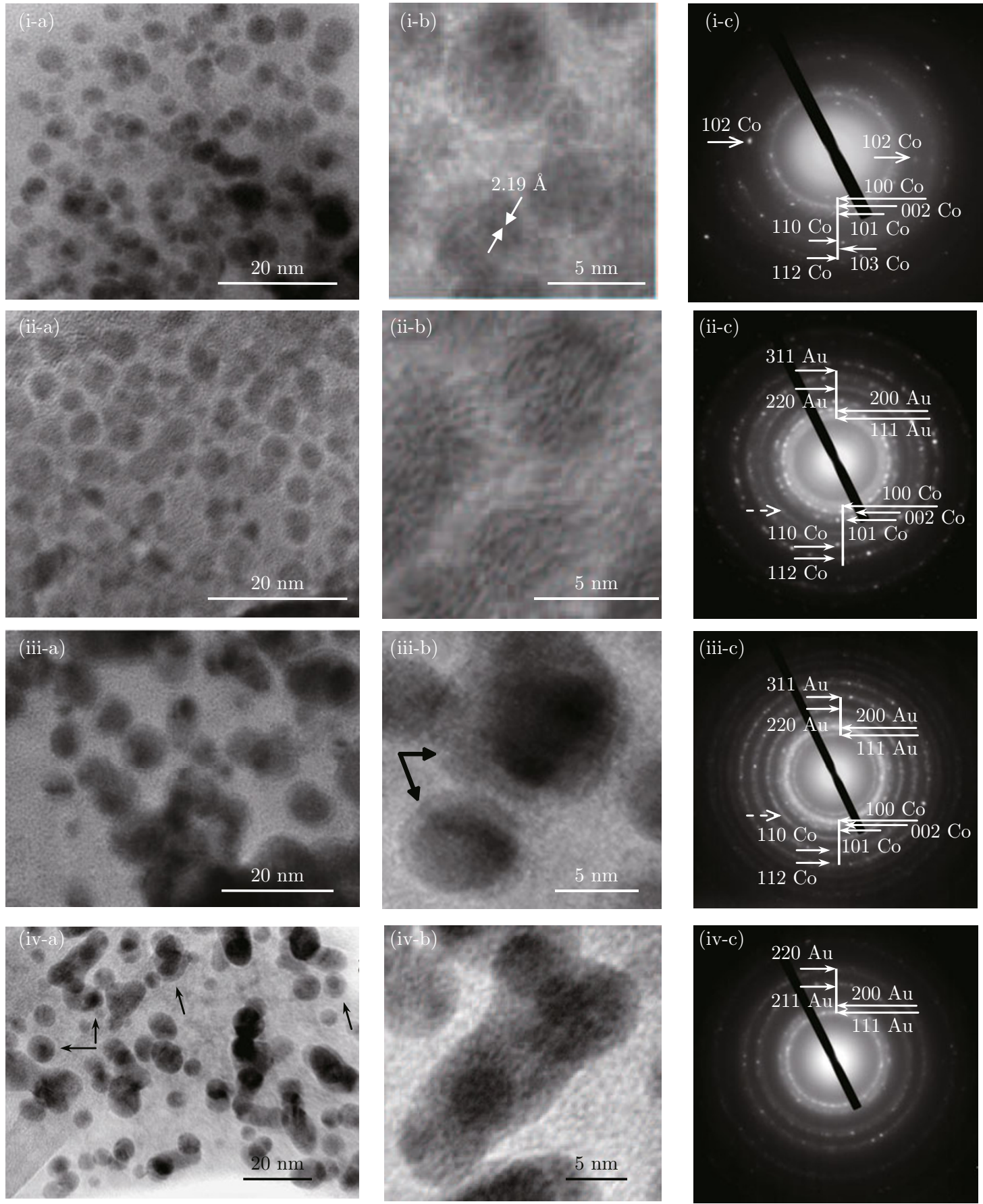

Fig. 1 (i-a) The wide view of the TEM image of the Co nanoparticles. (i-b) The magnified view of the TEM image of the specific Co nanoparticles. (i-c) The selected area electron diffraction pattern of the Co nanoparticles showing hexagonal close pack crystal structure. (ii-a) the wide-viewed TEM image of the Co@Au nanoparticles formed via the displacement method; (ii-b) the magnified TEM image of the specific Co@Au nanoparticles showing slightly lighter shells and darker cores; (ii-c) the selected area electron diffraction pattern of the Co@Au nanoparticles showing hexagonal close pack (hcp) Co crystal structure and face center cubic (fcc) Au crystal structure. (iii-a) the wide-viewed TEM image of the Co@Au nanoparticles formed via the displacement method and the first gold salt reduction process; (iii-b) the magnified TEM image of the specific Co@Au nanoparticles showing clearly lighter thick shells and darker cores; (iii-c) the selected area electron diffraction pattern of the Co@Au nanoparticles clearly showing hcp Co crystal structure and FCC Au crystal structure. (iv-a) the wide-viewed TEM image of the Co@Au nanoparticles formed via the displacement process and the second gold salt reduction process; (iv-b) the magnified TEM image of the specific Co@Au nanoparticles showing clearly lighter and thicker shells and darker cores; (iv-c) the selected area electron diffraction pattern of the Co@Au nanoparticles only showing fcc Au crystal structure due to their thick shell. 
the displacement methods (Fig. 1-ii-a), remaining similar to that of the starting Co NPs (but the averaged Co core is only $3.3 \mathrm{~nm}$ due to the displace of surface Co by $\mathrm{Au}$ ). From their SAED pattern, it is clear that there are $\mathrm{Au}$ atoms existing on these Co NPs, evidenced not only by those rings from hcp Co phase, but from fcc $\mathrm{Au}$ phase (Fig. 1-ii-c). The rings shown in Fig. 1-ii-c representing the [111], [200], [220], [311] plane of fcc Au phase give the d-spacing of $2.510 \AA, 2.151 \AA, 1.506 \AA$, $1.282 \AA$. Clearly, the ring for Co [002] almost overlaps with the ring for $\mathrm{Au}$ [200], the ring for Co [102] overlaps with the ring for $\mathrm{Au}$ [220], and the ring for Co [110] overlaps with the ring for $\mathrm{Au}$ [311].

Although the magnified images for several NPs show a slightly ordered atoms on the surface or edge of the NPs (Fig. 1-ii-b), no obvious Au crystal fringe or a continuous edge rim can be observed, as shown in the previous investigation $[16,23]$. The calculated shell thickness from the displacement of the $3.9 \mathrm{~nm}$ Co NPs formed from $0.16 \mathrm{~g} \mathrm{CoCl}_{2}$ with the $0.12 \mathrm{~g} \mathrm{KAuCl}_{4}$ is only 0.3 $\mathrm{nm}$, about 1 layer of $\mathrm{Au}$ atoms thick (radius of $\mathrm{Au}$ : $0.135 \mathrm{~nm}$ ). This observation indicates that this kind of layer is too thin to form a continuous $\mathrm{Au}$ shell, it only increases the roughness of surfaces of NPs after the Au atoms taking part of some surface sites of Co atoms to form some local Au clusters as evidenced by their clear SAED rings. This process is very similar as the etching or doped effect. These kinds of surfaces doped by $\mathrm{Au}$ atoms/clusters provide lots of active anchoring sites for further addition of $\mathrm{Au}$ atoms to form a thick and continuous Au shell.

As seen from Fig. 1-iii-a and b, the distinct variation in the contrast between the darker cores and the lighter edge rims in the NPs can be clearly observed after the further reduction-deposition of $\mathrm{Au}$ atoms on the Co@Au NPs formed by the displacement method, indicating continuous shells are formed on the surface of the cores $[16,23]$. Similar as the previous observation by Mandal et al. [16], the brighter edge rims are clearly made of Au shells since the mass contrast dominates over diffraction contrast in the TEM image, rendering the part in NPs formed by a larger atomic number element (i.e. $\mathrm{Au}$ ) lighter than that formed by a smaller atomic number element (i.e., Co) [16]. Rings from inner to outer in their SAED pattern that represent $\mathrm{Au}$ [111], Co [100]/Au [200], Co [002], Co [101], $\mathrm{Au}[220] / \mathrm{Co}$ [102], Au [311]/Co [110], Co [103], Co [102] planes further confirm that these NPs are made of Co and Au. Clearly, the ring for the Co [100] overlaps with the $\mathrm{Au}$ [200], the ring for the Co [102] (dash arrow) overlaps with the $\mathrm{Au}[220]$ and the ring for the $\mathrm{Co}$ [110] overlaps with $\mathrm{Au}$ [311]. Comparing with the SAED pattern for the Co@Au NPs formed by the displacement method, the ring intensities from $\mathrm{Au}$ shells become more distinguished than those from Co cores. A clearly-separated ring for $\mathrm{Au}$ [111], a stronger and wider ring for $\mathrm{Au}$ [200] and $\mathrm{Co}$ [100], and a stronger ring for $\mathrm{Au}[220]$ can be observed in the Co@Au NPs after the first reduction-deposition process (Fig. 1-iiic). The formed Co@Au NPs have an average size of $7.3 \pm 2.7 \mathrm{~nm}$ according to their TEM images. As observed from their TEM images, the Au shells become thick enough to be observed after the first reductiondeposition of additional Au on the surface of Co@Au NPs formed by the displacement method. The shell thickness is measured as $1.2 \pm 0.8 \mathrm{~nm}$, slightly smaller than the theoretical value $(2.1 \mathrm{~nm})$ due to the nonuniform core size and shell thickness distribution and the formation of some pure Au NPs. The formed pure $\mathrm{Au}$ NPs can be evidenced by the light pink top solution after the formed Co@Au NPs settle down by a magnet.

In order to increase the shell thickness and coverage, the reduction-deposition process was repeated once. As shown in Fig. 1-iv-a and b, some NPs indeed show relatively smaller cores and larger shells (particularly for those NPs marked by arrows). However, some small NPs without clear core-shell structures also appear. This result indicates that some $\mathrm{Au}$ atoms do not deposit on the surface the Co@Au NPs but self-grow to pure Au NPs. Simultaneously, clustering or aggregation of NPs becomes intensive probably due to the bridging effect from these small pure Au NPs to connect several Co@Au NPs together, as shown in Fig. 1-iv-b. Since the Au content in the whole NPs becomes dominant, the SAED pattern for these NPs only show features from Au shells and features of Co cores disappears completely (Fig. 1-iv-c). The measured size of NPs is $9.1 \pm 2.7 \mathrm{~nm}$ and the shell thickness is about $1.9 \pm$ 0.6. The calculated shell thickness should be about 2.5 $\mathrm{nm}$ after the second reduction-deposition. The reduced size and shell thickness is clearly caused by the formation of some pure Au NPs. Comparing with the $7.3 \pm$ $2.7 \mathrm{~nm}$ Co@Au NPs after the first reduction-deposition process, there is still additional $0.7 \mathrm{~nm}$ thick $\mathrm{Au}$ layer coated on the Co@Au NPs.

Comparing the SAED patterns for Co@Au NPs with different $\mathrm{Au}$ shell thicknesses (Fig. 1-ii-c, Fig. 1-iii-c, Fig. 1-iv-c), the ring intensities for Co parts decrease as the shell thickness increases. Finally, the SAED feature for the Co cores disappears completely when the shell becomes thick enough. Because the Co crystals have 3 crystal structures with only a slight difference in the formation energy [31], there may be some fine structure transitions (particularly the atom arrangement in the core-shell interface) caused by the Au shell thickness that cannot be revealed by their SAED patterns clearly. These fine structure transitions at nanoscale can crucially alter their electronic and magnetic properties of the core-shell NPs. Therefore, the fine structure transition driven by the shell thickness is further investigated by XANES and EXAFS based on Co K-edge measurement. 
Comparing the XANES spectra of the as-synthesized Co NPs (Fig. 2-A-i) and Co@Au NPs (Fig. 2-A-ii, iii, iv) with the standard $\mathrm{CoO}$ ( -325 mesh, Aldrich, Fig. 2$\mathrm{A}$-vi) and $\mathrm{Co}_{3} \mathrm{O}_{4}(<10 \mu \mathrm{m}$, Aldrich, Fig. 2-A-vii) powders, it turns out that they are free from oxidative impurities (or with ultra-thin oxidative layers) because
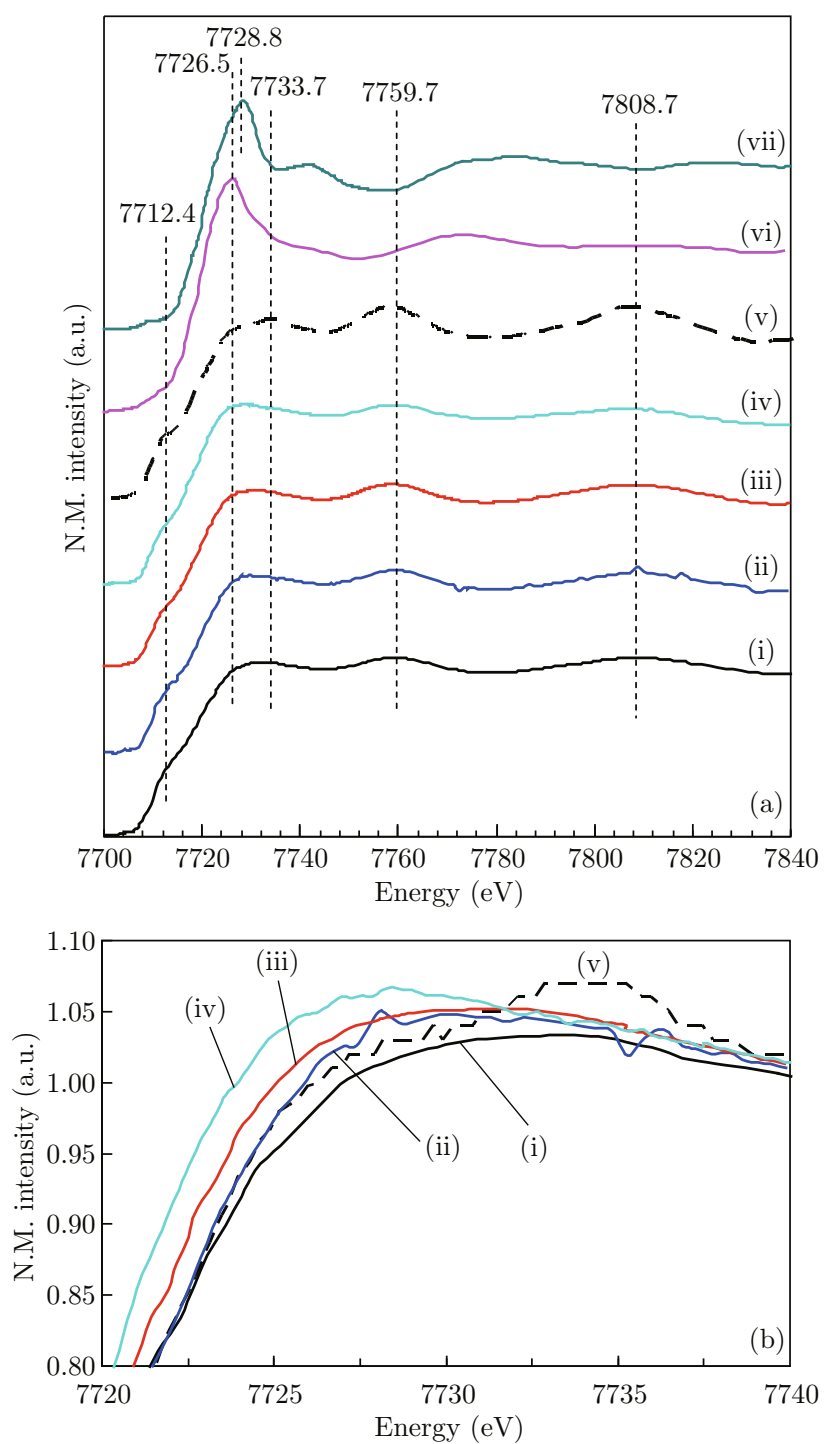

Fig. 2 (A) The X-ray absorption near K-edge structure (XANES) of (i) the Co nanoparticles; (ii) the Co@Au nanoparticles formed via the displacement process; (iii) the Co@Au nanoparticles formed via the displacement process and the first gold salt reduction process; (iv) the Co@Au nanoparticles formed via the displacement process and the second gold salt reduction process; (v) the standard hcp cobalt foil. (B) The magnified local XANES plotted at the same base line level of (i) the Co nanoparticles; (ii) the Co@Au nanoparticles formed via the displacement process; (iii) the Co@Au nanoparticles formed via the displacement process and the first gold salt reduction process; (iv) the $\mathrm{Co@Au} \mathrm{nanoparticles} \mathrm{formed} \mathrm{via} \mathrm{the} \mathrm{displacement} \mathrm{process}$ and the second gold salt reduction process; (v) the standard hcp cobalt foil; (vi) the standard $\mathrm{CoO}$ micro powder (325 mesh); (vii) the standard $\mathrm{Co}_{3} \mathrm{O}_{4}$ powder. neither significant chemical shift of the absorption edge to higher energy nor a systematically higher white line intensity, or a lower pre-edge intensity is observed [31]. Similar to the standard hcp Co foil, the clear pre-edge feature (the first inflection point at $7712.4 \mathrm{eV}$ ) and the presence of a shape resonance at about $7759.7 \mathrm{eV}$ indicate that one is dealing with the metallic Co phase [31, $35,38]$. Little magnetic bias observed in their hysteresis loops further confirms that these Co NPs and Co@Au NPs are free of oxidative impurities or the oxidative layers are too thin to be detected $[36,47]$. The much similar shape resonance feature (particularly in the white line at $7726.0 \mathrm{eV}$ and $7735.3 \mathrm{eV}$ ) as the hcp foil for the as-synthesized Co NPs suggest that they are mostly in the hcp phase, matching with their XRD and SAED characterization. However, as the coating thicknesses of Au shells are varied, significant shape resonance variations at the white line occur, featured by an increased intensity at around $7726.0 \mathrm{eV}$ (the first inflection) and a relatively reduced intensity at around $7735.3 \mathrm{eV}$. These variations can be more clearly observed if these curves are placed together (Fig. 2-B). Recalling the XANES spectra for our previous fcc and hcp Co NPs and the theoretical fcc Co and hcp Co phases [31, 35], it can be deduced that more fcc structural Co atoms exist in the Co@Au NPs with the thick shell, or some atoms in Co cores re-arrange from hcp-like to fcc-like structure as the $\mathrm{Au}$ shell thickness increases. This phenomenon can be partially explained by the electron negativity difference between cobalt (1.88) and gold (2.54). Since gold atoms attract electrons more than cobalt atoms, electrons in some Co atoms at the interface may be attracted to $\mathrm{Au}$ atoms, leading to Co atoms partially positive charged. As a consequence, the resonance intensity at $7726.0 \mathrm{eV}$ is increased, similar to the partially oxidization of cobalt [31]. However, this effect may partially contribute to the shape resonance change at the white line since the pre-edge inflection at $7712.4 \mathrm{eV}$ and the peak at $7759.7 \mathrm{eV}$ for metallic Co are still significant and no obvious magnetic bias can be observed in their hysteresis loops [36].

In order to elucidate the fine structure changes of the cobalt cores (or more fcc phased cobalt formation and the effect of $\mathrm{Co}-\mathrm{Au}$ bond formation) induced by some atoms transfer from hcp phase to fcc phase quantitatively, the theoretical XANES spectra of the Co mixtures with different ratios between the hcp phase and fcc phase can be performed by the simple linear addition of the theoretical XANES spectra of hcp phased Co (Fig. 3-i) and fcc phased Co (Fig. 3-ii) [31, 42, 43]. The matching is mainly based on the intensity ratios of the two main peaks between $7726.3-7727.3 \mathrm{eV}$ and at 7733.7-7734.7 eV at the white line of their XANES spectra. The matched XANES spectra for Co nanoparticles (Fig. 3-iii), Co@Au nanoparticles with shell thickness of $0.3 \mathrm{~nm}$ (Fig. 3-v), $1.2 \mathrm{~nm}$ (Fig. 3-vii) and 1.9 
nm (Fig. 3-ix) of the estimated fcc percentages by the theoretical simulation of their XANES spectra are summarized with their experimental spectra in Fig. 3. The calculation result indicates that the as-synthesized Co NPs are not mono-phase, which can be treated as a mixture of about $24 \%$ fcc phase and $76 \%$ hcp phase. With the formation of $\mathrm{Au}$ shell, the percentage of fccphased Co can be increased to 32\% for Co@Au NPs with $0.3 \mathrm{~nm}$ thick Au shell, and to $35 \%$ for Co@Au NPs with $1.2 \mathrm{~nm}$ thick Au shell, and then to $46 \%$ for Co@Au NPs with $1.9 \mathrm{~nm}$ thick Au shell. The increased fcc-phased Co percentages in the Co@Au NPs suggest that more hcp Co atoms transfer to fcc Co atoms with the shell thickness increase.

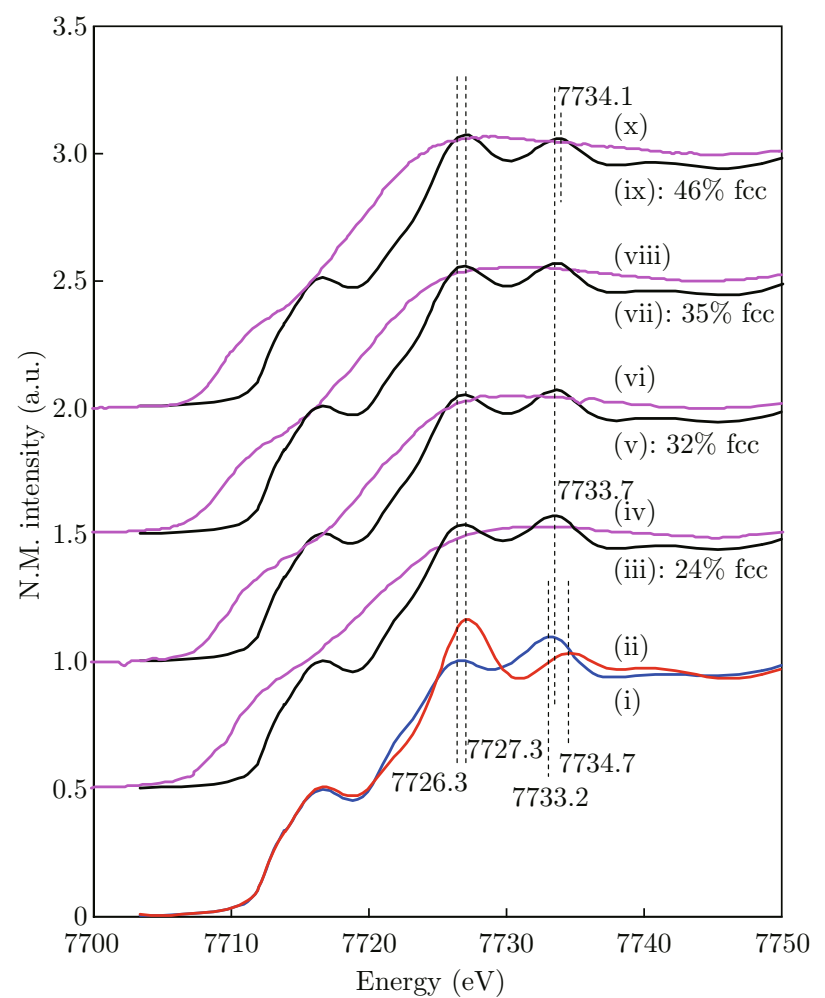

Fig. 3 Comparison of the theoretical XANES spectra for the Co nanoparticles with different percentages of fcc phased Co atoms (iii: $24 \%$; v: $32 \%$; vii: $35 \%$; ix: $46 \%$ ) calculated by the theoretical XANES spectra of hcp phased cobalt (i) and the theoretical fcc phased cobalt (ii) and the experimental results for the Co nanoparticles (iv), Co@Au nanoparticles with shell thickness of $0.3 \mathrm{~nm}$ (vi), $1.2 \mathrm{~nm}$ (viii) and $1.9 \mathrm{~nm}$ (x).

The EXAFS analysis on the average nearest-neighbor distance (R-spacing) gives the as-synthesized Co NPs (Fig. 4-i) with a mean inter-atom distance of $2.225 \AA$, longer than the standard hcp Co foil (2.178 $\AA$, Fig. 4$\mathrm{v}$ ), meaning that amorphous phased atoms, other phase Co atoms or crystal defects exist to dilate the interatom distance. The R-spacing of $\mathrm{Co}$ atoms decreases to $2.102 \AA$ (Fig. 4-ii) after the coating of Au shell by the

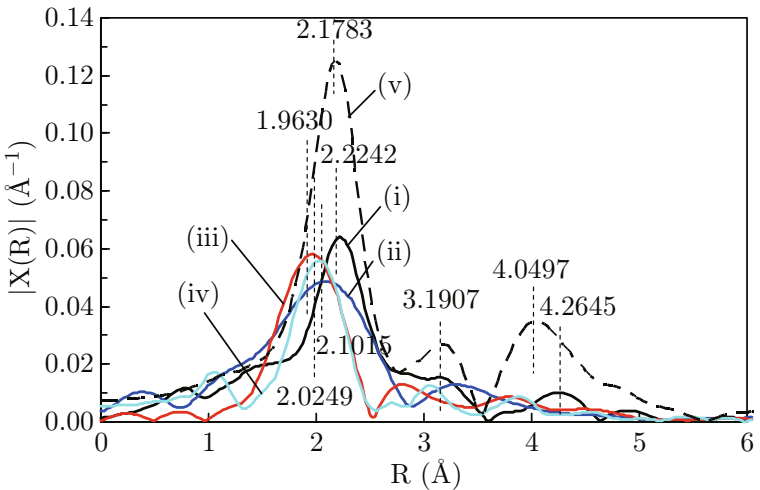

Fig. 4 The R-spacing of (i) the Co nanoparticles; (ii) the Co@Au nanoparticles formed via the displacement process; (iii) the Co@Au nanoparticles formed via the displacement process and the first gold salt reduction process; (iv) the $\mathrm{Co@Au} \mathrm{nanoparticles} \mathrm{formed} \mathrm{via} \mathrm{the} \mathrm{displacement} \mathrm{process}$ and the second gold salt reduction process; (v) the standard hcp cobalt foil after Extended X-ray absorption fine structure analysis.

displacement process, and then decreases to $1.963 \AA$ (Fig. 4-iii) after the first reduction-deposition of more $\mathrm{Au}$ atoms on the surface of the NPs. The reduced Rspacing has a similar trend as in our experimental and theoretical results on the R-spacing change from hcp phased Co to fcc phased Co [31], which is also consistent with the calculation results for Co mixtures with different contents of fcc phased atoms in Fig. 3. The considerable contraction in nearest-neighbor inter-atomic distance has also been observed by Baker et al. in $\mathrm{Fe} / \mathrm{Cu}$ and $\mathrm{Fe} / \mathrm{Au}$ nanoparticles with the shell formation [48]. Particularly, they also found that in the $\mathrm{Fe} / \mathrm{Cu}$ coreshell NPs, a $\mathrm{Cu}$ shell $\sim 20$ atom monolayers thick will cause the structure in the Fe core to switch from body centered cubic (bcc as in bulk Fe) to face centered cubic [48]. Therefore, our result suggests that the fcc phased $\mathrm{Au}$ atoms have a better compatibility (lattice matching) with the fcc phased Co than the hcp phased Co. This result indicates that fcc phased $\mathrm{Au}$ atoms have a higher compatibility with fcc phased Co than with hcp phased Co at nanoscale. The shell thickness driven local fine structure transition in the Co@Au NPs indicates that at least some atoms in the cobalt cores experiences a shift from hcp-like to fcc-like arrangement in order to match the fcc phased Au shell. When the shell thickness was further increased by a second reduction-deposition process, the R-spacing was increased slightly (Fig. 4iv) even though the calculation result suggests that more fcc phased atoms are in this sample (Fig. 3-ix). The slightly increased R-spacing in the final Co@Au NPs (Fig. 5-iv) can be explained by the formation of a significant amount of $\mathrm{Co}-\mathrm{Au}$ bonds during the second reduction-deposition process since the average nearestneighbor distance depend on the relative number of $\mathrm{Co}-\mathrm{Co}$ and $\mathrm{Co}-\mathrm{Au}$ bonds. Note: the $\mathrm{Co}-\mathrm{Co}, \mathrm{Co}-\mathrm{Au}$, and $\mathrm{Au}-\mathrm{Au}$ bond lengths in the lowest-energy struc- 
ture are $2.44 \AA, 2.61 \AA$, and $2.89 \AA$, respectively [49]. This phenomenon can also be attributed to the $\mathrm{Co}-\mathrm{Co}$ bond length expansion at the interface between the Co core and the Au shell due to the significant lattice mismatch of the two elements when the shell thickness is increased to a critical value [50]. As shown in Fig. 4-iii, the increased first inflection at $7726.0 \mathrm{eV}$ further indicates that the interaction between the Co atoms and $\mathrm{Au}$ atoms becomes stronger due to the formation of more $\mathrm{Co}-\mathrm{Au}$ bonds. When the shell becomes thicker, the reaction time will be long, which will favor to form more hcp or even epsilon Co according to the results in our previous article [31]. It is another evidence to confirm that the shell thickness affects the fine structure of the core materials by causing atomic re-arrangement between the hcp and the fcc structure, and forming Co$\mathrm{Au}$ bonds in the core-shell interface.

\section{Conclusions}

The Co@Au core shell nanoparticles with different shell thickness have been fabricated by a sequential microfluidic reactor. The shell thickness has significant effects on the fine structure of the core materials. Results of the analysis of the SAED, XANES and EXAFS of the Co@Au NPs with different Au thicknesses suggest that more atoms with fcc like arrangement can be formed in the Co@Au NPs with thick Au shells. This phenomenon can be explained by the combination of the slight difference in the formation energy between hcp and fcc structure in Co crystals and the formation of $\mathrm{Co}-\mathrm{Au}$ bonds at the interface.

\section{Acknowledgement}

The author appreciates the support from National Science Foundation of China (NSFC, Grant No. 50971010) and the Fundamental Research Funds for the Central Universities (YWF-11-03-Q-002).

\section{References}

[1] J. Garcia-Torres, E. Vallés and E. Gómez, J. Nanopart. Res. 12, 2189 (2010). http://dx.doi.org/10.1007/ s11051-009-9784-x

[2] A. M. Smith and S. Nie, Accounts Chem. Res. 43, 190 (2010). http://dx.doi.org/10.1021/ar9001069

[3] J. Zhang, Y. Tang, K. Lee and M. Ouyang, Nature 466, 91 (2010). http://dx.doi.org/10.1038/nature09150

[4] Z. Tang, Y. Song, Q. Sun, T. Zhang and Y. Jiang, Nanoscale 4, 386 (2012). http://dx.doi.org/10. 1039/c1nr10968e

[5] Y. Jin, C. Jia, S. W. Huang, M. O'Donnell and X. Gao, Nature Commun. 1, 41 (2010). doi:10.1038/ ncomms 1042
[6] Mansoureh Ganjali, Monireh Ganjali, Soraia Khoby and Mohammad Ali Meshkot, Nano-Micro Lett. 3, 256 (2011). http://dx.doi.org/10.3786/nml.v3i4. p256-263

[7] H. Kim, M. Achermann, L. P. Balet, J. A. Hollingsworth and V. I. Klimov, J. Am. Chem. Soc. 127, 544 (2005). http://dx.doi.org/10.1021/ ja047107x

[8] P. D. Cozzoli, T. Pellegrino and L. Manna, Chem. Soc. Rev. 35, 1195 (2006). http://dx.doi.org/10.1039/ b517790c

[9] J. Bai and J. P. Wang, Appl. Phys. Lett. 87, 152502 (2005). http://dx.doi.org/10.1063/1.2089171

[10] Y. J. Song, Z. Guo, H. M. O'Brian, J. F. Hormes and C. Kumar, Development of Magnetic Nanoparticles with Improved Properties, in: L. A. Broussard (Ed.) CAMD 2004 Annual Report, The J. Bennett Johnston, Sr., Center for Advanced Microstructures and Devices, 6980 Jefferson Highway, Baton Rouge, LA 70806, Baton Rouge, 2004, pp. 209-210.

[11] J. Schotter, O. Bethge, T. Maier and H. Brueckl, Appl. Phys. Lett. 93, 144105 (2008). http://dx.doi.org/ 10.1063/1.2992589

[12] Chandan Srivastava and K. V. L. Sushma, NanoMicro Lett. 4, 172 (2012). http://dx.doi.org/10. 3786/nml . v4i3.p172-175

[13] D. Farrell, Y. Cheng, R. W. McCallum, M. Sachan and S. A. Majetich, J. Phys. Chem. B 109, 13409 (2005). http://dx.doi.org/10.1021/jp050161v

[14] H. Zeng and S. Sun, Adv. Func. Mater. 18, 391 (2007). http://dx.doi.org/10.1002/adfm. 200701211

[15] Y. W. Jun, J. S. Choi and J. Cheon, Chem. Commun. 1203 (2007). http://dx.doi.org/10.1039/b614735f

[16] S. Mandal and K. M. Krishnan, J. Mater. Chem. 17, 372 (2007). http://dx.doi.org/10.1039/b613371c

[17] W. R. Lee, M. G. Kim, J. R. Choi, J. I. Park, S. J. Ko, S. J. Oh and J. Cheon, J. Am. Chem. Soc. 127, 16090 (2005). http://dx.doi.org/10.1021/ja053659j

[18] L. Wang, J. Luo, M. M. Maye, Q. A. Fan, R. Qiang, M. H. Engelhard, C. Wang, Y. Lin and C. J. Zhong, J. Mater. Chem. 15, 1821 (2005). http://dx.doi.org/ 10.1039/b501375e

[19] S. J. Cho, J. C. Idrobo, J. Olamit, K. Liu, N. D. Browning and S. M. Kauzlarich, Chem. Mater. 17, 3181 (2005). http://dx.doi.org/10.1021/cm0500713

[20] Y. Bao, H. Calderon and K. M. Krishnan, J. Phys. Chem. C 111, 1941 (2007). http://pubs.acs.org/ doi/abs/10.1021/jp066871y

[21] K. J. Carroll, D. M. Hudgins, S. Spurgeon, K. M. Kemner, B. Mishra, M. I. Boyanov, L. W. Brown III, M. L. Taheri and E. E. Carpenter, Chem. Mater. 22, 6291 (2010). http://dx.doi.org/10.1021/cm101996u

[22] Y. Xu and J. P. Wang, IEEE Trans. Magn. 43, 3109 (2007). http://dx.doi.org/10.1109/TMAG. 2007.894006

[23] C. S. Levin, C. Hofmann, T. A. Ali, A. T. Kelly, E. Morosan, P. Nordlander, K. H. Whitmire and N. J. Halas, ACS Nano 3, 1379 (2009). http://dx.doi.org/ $10.1021 / \mathrm{nn} 900118 \mathrm{a}$ 
[24] S. H. Baker, M. Roy, S. C. Thornton, M. Qureshi and C. Binns, J. Phys.: Condens Mat. 22, 5301 (2010). http://dx.doi.org/10.1088/0953-8984/22/ $38 / 385301$

[25] Y. Song, L. L. Henry and W. T. Yang, Langmuir 25, 10209 (2009). http://dx.doi.org/10.1021/ 1 a9009866

[26] T. L. Sounart, P. A. Safier, J. A. Voigt, J. Hoyt, D. R. Tallant, C. M. Matzke and T. A. Michalske, Lab on a chip 7, 908 (2007). http://dx.doi.org/10.1039/ b703810k

[27] A. J. deMello, Nature 442, 394 (2006). http://dx. doi.org/10.1038/nature05062

[28] Y. Song and L. L. Henry, Nanoscale Res. Lett. 4, 1130 (2009). http://dx.doi.org/10.1007/ s11671-009-9369-8

[29] S. He, T. Kohira, M. Uehara, T. Kitamura, H. Nakamura, M. Miyazaki and H. Maeda, Chemistry Letters 34, 748 (2005). http://dx.doi.org/10.1246/cl. 2005.748

[30] B. Zhao, J. S. Moore and D. J. Beebe, Science 291, 1023 (2001). http://dx.doi.org/10.1126/science. 291.5506.1023

[31] Y. Song, H. Modrow, L. L. Henry, C. K. Saw, E. E. Doomes, V. Palshin, J. Hormes and C. S. S. R. Kumar, Chem. Mater. 18, 2817 (2006). http://dx.doi. org/10.1021/cm052811d

[32] Y. Song, P. Jin and T. Zhang, Mater. Lett. 64, 1789 (2010). http://dx.doi.org/10.1016/j.matlet. 2010.05 .025

[33] Yujun Song, R. Li, Q. Sun and P. Jin, Chem. Eng. J. 168, 477 (2011). http://dx.doi.org/10.1016/j.cej. 2011.01 .059

[34] S. Krishnadasan, J. Tovilla, R. Vilar, A. J. deMello and J. C. deMello, J. Mater. Chem. 14, 2655 (2004). http://dx.doi.org/10.1039/b401559b

[35] Y. Song, T. Zhang, W. T. Yang, S. Albin and L. L. Henry, Crystal Growth Des. 8, 3766 (2008). http:// dx.doi.org/10.1021/cg8003992

[36] Y. Song, J. Ding and Y. Wang, J. Phys. Chem. C 116, 11343 (2012). http://dx.doi.org/10.1021/ jp300118z

[37] Y. Song, C. S. S. R. Kumar and J. Hormes, J. Micromech. Microeng 14, 932 (2004). http://dx.doi. org/10.1088/0960-1317/14/7/013
[38] Y. Song, E. E. Domes, J. Prindle, R. Tittsworth, J. Hormes and C. S. S. R. Kumar, J. Phys. Chem. B 109, 9330 (2005). http://dx.doi.org/10.1021/jp044777g

[39] Y. Song, S. Sun, T. Zhang, P. Jin and L. Han, J. Nanopart. Res. 12, 2689 (2010). http://dx.doi.org/ 10.1007/s11051-010-0012-5

[40] Y. Song, C. S. S. R. Kumar and J. Hormes, J. Nanosci. Nanotech. 4, 788 (2004). http://dx.doi. org/10.1166/jnn.2004.111

[41] H. Bonnemann, G. Braun, W. Brijoux, R. Brinkmann, A. T. Schulze, K. Seevogel and K. Siepen, J. Organomet. Chem. 520, 143 (1996). http://dx.doi. org/10 . 1016/0022-328X (96) 06273-0

[42] D. E. Sayers, Report of the International XAFS Society Standards and Criteria Committee, in: the IXS Standards and Criteria Committee (2000). http://ixs.iit.edu/subcommittee_reports/sc/ sc00report.pdf

[43] The IXS Standards and Criteria Committee, Error Reporting Recommendations: A Report of the Standards and Criteria Committee (2000). http://ixs.iit.edu/ subcommittee_reports/sc/err-rep.pdf

[44] V. Skumryev, S. Stoyanov, Y. Zhang, Hadjipanayis, G. D. Givord and J. Nogues, Nature 423, 850 (2003). http://dx.doi.org/10.1038/nature01687

[45] A. H. Lu, E. L. Salabas and F. Schuth, Angew. Chem. Int. Ed. 46, 1222 (2007). http://dx.doi.org/ 10.1002/anie. 200602866

[46] C. Petit, Z. L. Wang and M. P. Pileni, J. Phys. Chem. B 109, 15309 (2005). http://dx.doi.org/10.1021/ jp052487+

[47] T. Wen and K. M. Krishnan, J. Appl. Phys. 109, 07B515 (2011). http://dx.doi.org/+10.1063/1. 3544493

[48] M. R. S H Baker, S C Thornton,M Qureshi and C Binns, J. Phys.: Condens Mat. 22, 385301 (2010). http://dx.doi.org/10.1088/0953-8984/22/ $38 / 385301$

[49] J. Wang, J. Bai, J. Jellinek and X. C. Zeng, J. Am. Chem. Soc. 129, 4110 (2007). http://dx.doi.org/10. 1021/ja0664234

[50] P. Kluth, B. Hoy, B. Johannessen, S. G. Dunn, G. J. Foran and M. C. Ridgway, Appl. Phys. Lett. 89, 153118 (2006). http://dx.doi.org/10.1063/1.2360891 